

\title{
Self-organized flocking with a mobile robot swarm: a novel motion control method
}

Eliseo Ferrante, Ali Emre Turgut, Cristián Huepe, Alessandro Stranieri, Carlo Pinciroli, Marco Dorigo

\section{To cite this version:}

Eliseo Ferrante, Ali Emre Turgut, Cristián Huepe, Alessandro Stranieri, Carlo Pinciroli, et al.. Selforganized flocking with a mobile robot swarm: a novel motion control method . Adaptive Behavior, 2012, 20 (6), pp.460-477. 10.1177/1059712312462248 . hal-01406049

\section{HAL Id: hal-01406049 \\ https://hal.science/hal-01406049}

Submitted on 6 Dec 2016

HAL is a multi-disciplinary open access archive for the deposit and dissemination of scientific research documents, whether they are published or not. The documents may come from teaching and research institutions in France or abroad, or from public or private research centers.
L'archive ouverte pluridisciplinaire HAL, est destinée au dépôt et à la diffusion de documents scientifiques de niveau recherche, publiés ou non, émanant des établissements d'enseignement et de recherche français ou étrangers, des laboratoires publics ou privés. 


\section{Université Lỉbre de Bruxelles}

Self-Organized Flocking with a Mobile Robot Swarm: a Novel Motion Control Method

E. Ferrante, A. E. Turgut, C. Huepe, A. Stranieri, C. Pinciroli, and M. Dorigo

\section{IRIDIA - Technical Report Series}

Technical Report No.

TR/IRIDIA/2012-003

March 2012

Last revision: May 2013 


\section{IRIDIA - Technical Report Series}

ISSN 1781-3794

Published by:

IRIDIA, Institut de Recherches Interdisciplinaires

et de Développements en Intelligence Artificielle

Université LiBRE DE BRUXELles

Av F. D. Roosevelt 50, CP 194/6

1050 Bruxelles, Belgium

Technical report number TR/IRIDIA/2012-003

Revision history:

TR/IRIDIA/2012-003.001 March 2012

TR/IRIDIA/2012-003.002 May 2012

TR/IRIDIA/2012-003.003 May 2013

The information provided is the sole responsibility of the authors and does not necessarily reflect the opinion of the members of IRIDIA. The authors take full responsibility for any copyright breaches that may result from publication of this paper in the IRIDIA Technical Report Series. IRIDIA is not responsible for any use that might be made of data appearing in this publication. 


\title{
Self-Organized Flocking with a Mobile Robot Swarm:
}

\author{
a Novel Motion Control Method \\ Eliseo Ferrante ${ }^{12}$, Ali Emre Turgut ${ }^{12}$, Cristián Huepe ${ }^{3}$, \\ Alessandro Stranieri ${ }^{1}$, Carlo Pinciroli ${ }^{1}$ and Marco Dorigo ${ }^{1}$ \\ ${ }^{1}$ IRIDIA, CoDE, Université Libre de Bruxelles,
} 50 Av. Franklin Roosevelt CP 194/6, 1050 Brussels, Belgium

${ }^{2}$ Laboratory of Socioecology and Social Evolution, Katholieke Universiteit Leuven, 59 Naamsestraat - bus 2466, 3000 Leuven, Belgium

${ }^{3}$ CHuepe Labs Inc., 614 N Paulina St, Chicago IL 60622, USA

Received: date / Accepted: date

Running title: A novel motion control method for flocking

Contact person: Eliseo Ferrante

Laboratory of Socioecology and Social Evolution

Naamsestraat 59, B-3000 Leuven, Belgium

E-mail: eliseo.ferrante@bio.kuleuven.be

Phone: +32-488-27-68-42 


\begin{abstract}
In flocking, a swarm of robots moves cohesively in a common direction. Traditionally, flocking is realized using two main control rules: proximal control, which controls the cohesion of the swarm using local range-and-bearing information about neighboring robots; and alignment control, which allows the robots to align in a common direction and uses more elaborate sensing mechanisms to obtain the orientation of neighboring robots. So far, limited attention has been given to motion control, used to translate the output of these two control rules into robot motion.

In this paper, we propose a novel motion control method: magnitude-dependent motion control (MDMC). Through simulations and real robot experiments, we show that, with MDMC, flocking in a random direction is possible without the need of alignment control and of robots having a preferred direction of travel. MDMC has the advantage to be implementable on very simple robots that lack the capability to detect the orientation of their neighbors. Additionally, we introduce a small proportion of robots informed about a desired direction of travel. We compare MDMC with a motion control method used in previous robotics literature, which we call magnitudeindependent motion control (MIMC), and we show that the swarms can travel longer distances in the desired direction when using MDMC instead of MIMC. Finally, we systematically study flocking under various conditions: with or without alignment control, with or without informed robots, with MDMC or with MIMC.
\end{abstract}

Keywords: Flocking, swarm robotics, swarm intelligence, coordinated motion, self-organization 


\section{Introduction}

Flocking, also referred to as self-organized flocking, is a collective behavior that is widely observed in nature. It can be defined as the cohesive and ordered motion of a group of individuals in a common direction. Examples can be seen in many animal species, from locusts to birds and quadrupeds.

One of the first computer models of the flocking behavior was proposed by Reynolds (1987). In his work, the behavior of each individual of the swarm is determined by three components: attraction, repulsion and alignment. The attraction component allows the individual to be attracted to its neighbors to keep the group together. The repulsion component allows the individual to stay away from its neighbors to avoid collisions. The alignment component allows the individual to match its heading direction to an average of the heading of its neighbors, thus making all individuals point in the same direction. Note that these three components had already been introduced in a previous flocking model by Suzuki and Sakai (1973).

Reynolds' model can be considered as a seminal work that inspired much subsequent literature. In the majority of these later works, none of the individuals composing the swarm has a preferred direction of motion. This literature includes works in biology (Couzin et al., 2002), statistical physics (Vicsek et al., 1995) and robotics (Turgut et al., 2008a). Another part of the literature followed the seminal work of Couzin et al. (2005), who introduced the notions of informed individuals, which have a preferred direction of motion or goal direction, and non-informed individuals, which are not aware of the goal direction.

In robotics, all works use rules of attraction and repulsion that are similar to those studied by Reynolds. However, only some works use the alignment rule. Turgut et al. (2008a) developed a flocking method consisting of proximal control, which encodes the attraction and repulsion rules, and alignment control, which encodes the alignment rule. Turgut et al. (2008a) achieved a flocking behavior on autonomous robots that, for the first time, did not require external computation or communication devices. They did not consider informed robots, but these were later introduced in a subsequent study by Çelikkanat and Şahin (2010). Other works achieved alignment either by using global information or simulated sensors (Hayes and Dormiani-Tabatabaei, 2002; Holland et al., 2005) or by making all or most of the robots aware of the desired goal direction (Matarić, 1994; Spears et al., 2004). 
When the knowledge of the goal direction is widely available in the swarm, the alignment of all robots in the same direction results merely from this global information rather than from a self-organization process.

In this paper, we take a perspective that is different from what is found in the literature. We propose magnitude-dependent motion control (MDMC), a method that, together with proximal control, achieves flocking with minimal components. By minimal we mean that, differently from Turgut et al. (2008a), alignment control is not needed; differently from (Hayes and Dormiani-Tabatabaei, 2002; Holland et al., 2005), external hardware is not needed; differently from (Matarić, 1994; Spears et al., 2004), information about the goal direction is not needed. Our method achieves ordered and cohesive flocking using very simple robot hardware: robots only need to be non-holonomic and to sense their neighbors' range and bearing. Using this method, large swarms composed of hundreds or thousands of even very simple robots (such as nano-robots, aerial robots or robots used for extra-planetary missions) could be used to explore large environments. Furthermore, we believe that our model is simple but powerful enough to be used as a reference model for biology, for studying the movement of very simple animals (Simpson et al., 2006) or cells (Szabó et al., 2006) and, in the context of statistical physics, for studying the principles underlying swarms of active particles (Vicsek et al., 1995; Romanczuk et al., 2009).

The contribution of this paper is twofold. First, we introduce MDMC, a method capable of achieving self-organized flocking without alignment control and without informed robots. Second, we systematically study self-organized flocking by performing extensive simulations and validating our results with real robot experiments. The study is conducted with and without alignment control, with and without informed robots, with MDMC and with magnitude-independent motion control (MIMC) (Turgut et al., 2008a). We show that MDMC always outperforms MIMC when alignment control is not used, both with and without informed robots.

The rest of the paper is organized as follows. In Section 2, we introduce the flocking and the motion control methods. In Section 3, we explain how the model has been implemented on the utilized robotics platform. In Section 4, we describe the metrics, the simulation and the real robot experimental setup. In Section 5 and in Section 6, we present the 
results obtained without and with informed robots, respectively. In Section 7, we discuss related works within robotics literature and we also survey some related works in statistical physics. Finally, in Section 8 we conclude and we discuss possible future work.

\section{Self-organized Flocking}

In this section we describe the method we use to achieve flocking. The method is made of different components: proximal control, alignment control and motion control. Motion control is, in turn, realized either with the novel MDMC or with MIMC.

The overall flocking method is defined as follows. We assume that the robot under consideration, the focal robot, computes a flocking control vector denoted by $\mathbf{f}$. The expression of the flocking control vector, in the most complete form that we call Model 1 , is:

$$
\mathbf{f}=\mathbf{p}+\mathbf{a}+\mathbf{g}
$$

where $\mathbf{p}$ is the proximal control vector, encoding the attraction and repulsion rules, $\mathbf{a}$ is the alignment control vector, encoding the alignment rule, and $\mathbf{g}$ is the goal direction vector, encoding the goal following behavior used only by the informed robots. In this study we will also consider the following models:

$$
\begin{gathered}
\mathbf{f}=\mathbf{p}, \\
\mathbf{f}=\mathbf{p}+\mathbf{g}, \\
\mathbf{f}=\mathbf{p}+\mathbf{a} .
\end{gathered}
$$

In Section 4, we explain how these models are utilized. 


\subsection{Proximal control}

The proximal control part of the method is common to all four models. The main idea of proximal control is that, in order to achieve cohesive flocking, a robot needs to keep a certain distance from its neighbors. The proximal control vector encodes the attraction and repulsion rules: a robot tends to move closer to its neighbors when their distance is greater than the desired distance and tends to move away from them when their distance is smaller than the desired distance.

Proximal control assumes that the focal robot is able to sense the range and bearing of its neighboring robots within a maximum interaction distance $D_{p}$. The proximal control vector $\mathbf{p}$ is computed as:

$$
\mathbf{p}=\sum_{i=1}^{m_{p}} p_{i}\left(d_{i}\right) e^{j \phi_{i}}
$$

where $p_{i}\left(d_{i}\right) e^{j \phi_{i}}$ is a vector expressed in the complex plane; $m_{p}$ is the number of neighboring robots perceived by the focal robot within range $D_{p}$; and $d_{i}$ and $\phi_{i}$ denote respectively the relative range and bearing of the $i^{\text {th }}$ neighboring robot, expressed in the body-fixed reference frame of the focal robot $^{1}$. The magnitude of the vector, $p_{i}\left(d_{i}\right)$, is computed as:

$$
p_{i}\left(d_{i}\right)=-\frac{\partial P\left(d_{i}\right)}{\partial d_{i}}=-\frac{4 \alpha \epsilon}{d_{i}}\left[2\left(\frac{\sigma}{d_{i}}\right)^{2 \alpha}-\left(\frac{\sigma}{d_{i}}\right)^{\alpha}\right] .
$$

In words, $p_{i}\left(d_{i}\right)$ is defined as the negative derivative of the virtual potential function $P\left(d_{i}\right)$. A virtual potential function is often used in swarm robotics and statistical physics to model social interaction between robots (Spears et al., 2004) or particles (Gregoire et al., 2003). In our study, we use a generalized version of the Lennard-Jones potential function:

$$
P_{i}\left(d_{i}\right)=4 \epsilon\left[\left(\frac{\sigma}{d_{i}}\right)^{2 \alpha}-\left(\frac{\sigma}{d_{i}}\right)^{\alpha}\right] .
$$

Parameter $\epsilon$ determines the depth of the potential function; that is, the minimum of the potential function located at $d_{i}=d_{d e s}=2^{\frac{1}{\alpha}} \sigma$ is $-\epsilon$. We generalized the standard LennardJones potential used in (Hettiarachchi and Spears, 2009) in order to have the flexibility to choose the exponent $\alpha$, which determines the steepness of the function. Originally, Lennard-Jones set $\alpha=6$. In this paper, as summarized in Table 1 , we use $\alpha=2$, which

\footnotetext{
${ }^{1}$ The body-fixed reference frame is fixed to the center of a robot, its $x$-axis points to the front of the robot and its $y$-axis is coincident with the rotation axis of the wheels.
} 
corresponds to a lower steepness, as this value was found to achieve better performance in experiments with real robots.

Finally, regarding the maximum interaction distance $D_{p}$, in general it can be less than or equal to the maximum sensing range of the sensor. In this study, in order for the proposed model to work in the absence of alignment control and informed robots, we found that $D_{p}$ must be chosen so that, when the swarm reaches a stable formation, interactions among robots are limited to the first neighbors only in the Voronoi sense (Voronoi, 1908). In order to achieve this without having to compute the Voronoi tesselation, we set $D_{p}=$ $1.8 \cdot 2^{\frac{1}{6}} \sigma$, that is, the maximum interaction range is less than twice the desired distance $2^{\frac{1}{6}} \sigma$ between two robots.

\subsection{Alignment Control}

The main idea of alignment control is that a robot computes the average orientation of its neighbors and adjusts its own orientation accordingly.

The method used for alignment control uses the following equation:

$$
\mathbf{a}=\frac{\sum_{i=0}^{m_{a}} e^{j \theta_{i}}}{\| \sum_{i=0}^{m_{a}} e^{j \theta_{i} \|}}
$$

where $\theta_{0}$ is the orientation of the focal robot, $m_{a}$ is the number of neighbors within a range $D_{a}$ of the focal robot, $\theta_{i}, i \in\left\{1, \ldots, m_{a}\right\}$ are the orientation of the neighbors expressed in the body-fixed reference frame of the focal robot and $\|\cdot\|$ denotes the length of a vector. The focal robot calculates its alignment control vector as the average orientation of the $m_{a}$ neighboring robots and of its own orientation (which we label as $i=0$ ).

\subsection{Motion control}

Motion control is used to convert the flocking control vector $\mathbf{f}$ into the forward speed $u$ and the angular speed $\omega$ of the robot that is assumed to be non-holonomic. Below, we describe the two motion control methods considered in this paper. 


\subsubsection{Magnitude-dependent motion control (MDMC)}

MDMC is the novel contribution of this paper. In MDMC, the forward and angular speed of the robot depend on both the magnitude and the direction of the flocking control vector. Therefore, the method uses all the information contained in this vector.

We define MDMC as follows. Let $\mathbf{f}_{x}$ and $\mathbf{f}_{y}$ denote the projection of the flocking control vector (f) on the $x$ - and $y$-axis of the body-fixed reference frame, respectively. The forward speed $u$ is directly proportional to the $x$ component of the vector, pointing in the same direction as the robot's orientation. The angular speed $\omega$, instead, is directly proportional to the $y$ component of the vector, pointing in the direction perpendicular to the robot's orientation. The corresponding equations are

$$
\begin{gathered}
u=K_{1} \mathbf{f}_{x}+U ; \\
\omega=K_{2} \mathbf{f}_{y},
\end{gathered}
$$

where $U$ is a forward biasing speed, and $K_{1}$ and $K_{2}$ are the linear and angular gains, respectively.

The rationale of this method is the following: the larger the $x$-component of the flocking control vector, the faster we want the robot to move forward; the larger the $y$-component of the flocking control vector, the faster we want the robot to turn. Note that, with MDMC, robots can in principle move also backwards, but they have a propension to move forward due to the presence of the $+U$ term in the equation for the forward speed.

\subsubsection{Magnitude-independent motion control (MIMC)}

MIMC was introduced for the first time in Turgut et al. (2008a) and later used in subsequent work by Çelikkanat and Şahin (2010). In MIMC, the forward and angular speed of the robot only partially depend on the information contained in the flocking control vector. By "partially" we mean that, for calculating the speeds, only the direction of the flocking control vector is taken into account, while the magnitude is discarded.

Let $\mathbf{o}$ be the unit vector matching the orientation of the focal robot (it points forward along the $x$-axis of the body-fixed reference frame). The forward velocity and the angular 
velocities are computed as:

$$
\begin{gathered}
u= \begin{cases}\left(\mathbf{o} \cdot \frac{\mathbf{f}}{\|\mathbf{f}\|}\right) U, & \text { if } \mathbf{o} \cdot \frac{\mathbf{f}}{\|\mathbf{f}\|} \geq 0 \\
0, & \text { otherwise; }\end{cases} \\
\omega=K_{3}(\angle \mathbf{o}-\angle \mathbf{f}),
\end{gathered}
$$

where $K_{3}$ is the angular gain and - denotes the dot product of two vectors.

The rationale of the method is the following. The robot modulates its forward speed according to the angular difference between the flocking control vector and its orientation. When the two directions are aligned, the robot moves at maximum speed $U$. When the angular difference is equal or larger than 90 degrees, the robot does not move forward but rotates on the spot. In between these two cases, the forward speed is interpolated between 0 and $U$ according to the dot product between the flocking control vector and the robot's orientation. The angular speed is instead proportional to the angular difference between the flocking control vector and the robot's orientation. Note that, with MIMC, the robots are not able to move backwards.

\section{Self-organized Flocking with Mobile Robots}

The mobile robot we use is the foot-bot (Bonani et al., 2010), shown in Figure 1a. The foot-bot is commonly used for swarm robotics experiments and was developed during the Swarmanoid project ${ }^{2}$ (Dorigo et al., in press).

\subsection{The hardware}

We utilized the following sensors and actuators: i) A light sensor, used to measure the intensity and the direction of the light in the environment. ii) A range and bearing system (RAB), used to measure the range and the bearing of the other robots. iii) Two wheel actuators, used to control independently the speed of the left and the right wheels of the robot.

\footnotetext{
${ }^{2}$ http://www. swarmanoid.org/
} 


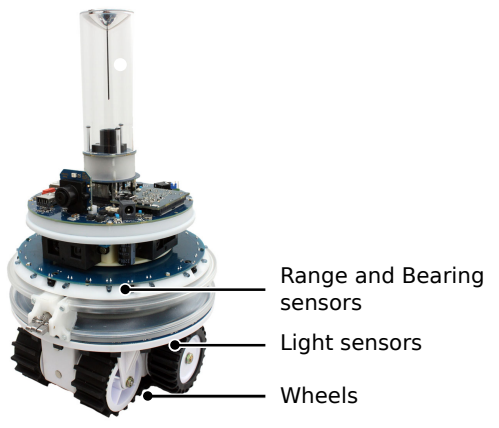

(a)

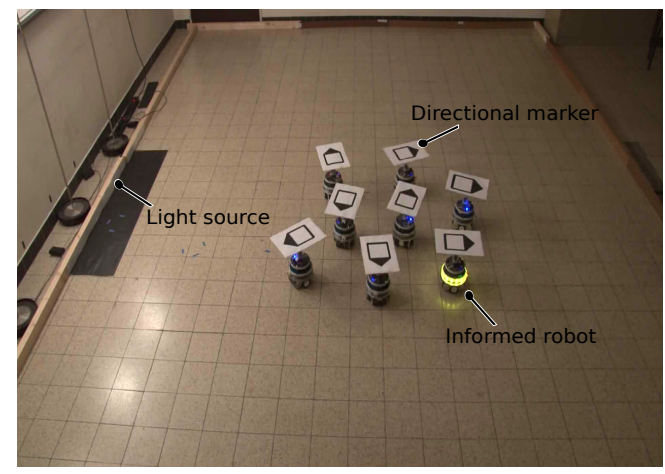

(b)

Figure 1: (a) The foot-bot with its used sensors and actuators. (b) The arena as seen by the overhead camera used for tracking: on the left side we created a light source by placing four lamps; a cardboard hat with a directional marker is placed on each foot-bot, in order to detect its orientation to compute the required metrics; the glowing robots are informed about the desired goal direction (this example is taken from experiments with informed robots). Note that LEDs and cardboard hats are used for debugging, visualization and measurement purposes only, but are never utilized by the controller.

\subsection{The simulator}

We used the ARGoS ${ }^{3}$ simulator (Pinciroli et al., 2011) to execute physics-based simulations of the robot dynamics when real-robot experiments are not possible or practical. ARGoS is an open-source, plug-in based simulator in which custom-made physics engines and robots with any desired degree of accuracy can be added. We used a custom-made 2D-kinematic physics engine and a realistic model of the foot-bot that simulates very accurately all the sensors and actuators. Another feature of ARGoS is the possibility to cross-compile controllers both in simulation and on the real robots. This allowed us to seamlessly port the same controller studied in simulation to the real robots.

\subsection{Flocking implementation}

The method described in Section 2 is implemented both on simulated and on real robots as follows. Proximal control is achieved by using the RAB to measure the relative range and bearing $d_{i}$ and $\phi_{i}$ of the $i_{t h}$ neighbor. Since robots are not able to measure the orientation of their neighbors directly, we achieve alignment control by using a method similar to the one in Turgut et al. (2008a). A light source, placed in a fixed position in the environment,

\footnotetext{
${ }^{3}$ http://iridia.ulb.ac.be/argos/
} 
is used to define a reference frame common to all robots. Each aligning robot measures its orientation $\theta_{0}$ with respect to this reference frame by using its on-board light sensor. It then communicates $\theta_{0}$ to its neighbors by using the communication unit present in the RAB. The result is equivalent to having neighbors that are able to sense each others' orientations. Only the informed robots are given the goal direction, expressed with respect to the fixed light source ${ }^{4}$.

To achieve motion control, we first limit the forward speed to the interval $[-U, U]$, and the angular speed to the interval $[-\Omega, \Omega]$. We then use the differential drive model used in (Turgut et al., 2008a) to convert the forward speed $u$ and the angular speed $\omega$ into the linear speeds of the left $\left(N_{L}\right)$ and right $\left(N_{R}\right)$ wheel:

$$
\begin{aligned}
& N_{L}=\left(u+\frac{\omega}{2} l\right), \\
& N_{R}=\left(u-\frac{\omega}{2} l\right),
\end{aligned}
$$

where $l$ is the distance between the wheels. Note that, in MIMC and MDMC, we fix the maximum attainable speeds to the same values. This is done for two reasons: first, in order to be able to properly compare the performance of the two methods; second, in order to have numerically stable simulation results with the control time-step used ( $\Delta t=0.1 \mathrm{~s})$, which requires limiting both speeds to low values.

We summarize the values of the parameters used in our experiments in Table 1 (simulation and controller parameters) and Table 2 (real robot). Note that the parameters used for the controller in simulation are the same as those used in the real robot experiments.

\section{Experimental setup}

In this section, we introduce the tools and the settings used in the experiments. First, we define the metrics used to assess the performance. Then, we describe the experimental setup used in the simulations and with the real robots.

\footnotetext{
${ }^{4}$ Note that non-informed robots that do not use alignment control do not need the light sensor. Therefore, the light was not used in experiments without informed robots and without alignment control.
} 


\begin{tabular}{|c|l|c|}
\hline Parameter & Description & Value \\
\hline \hline$N$ & Number of robots & $\{10,50, \mathbf{1 0 0}, 500,1000\}$ \\
\hline$\rho$ & Proportion of informed robots & $\{\mathbf{0 . 0 1}, 0.05,0.1,0.15,0.2\}$ \\
\hline$U$ & Maximum forward speed & $0.005 \mathrm{~m} / \mathrm{s}$ \\
\hline$\Omega$ & Maximum angular speed & $0.5 \mathrm{mad} / \mathrm{s}$ \\
\hline$K_{1}$ & MDMC linear gain & $0.06 \mathrm{rad} / \mathrm{s}$ \\
\hline$K_{2}$ & MDMC angular gain & $0.25 \mathrm{rad} / \mathrm{s}$ \\
\hline$K_{3}$ & MIMC angular gain & $0.14 \mathrm{~m}$ \\
\hline$l$ & Inter-wheel distance & 2 \\
\hline$\alpha$ & Steepness of potential function & 1.5 \\
\hline$\epsilon$ & Strength of potential function & $0.45 \mathrm{~m}$ \\
\hline$d_{d e s}$ & Desired inter-robot distance & $1.8 d_{d e s}=0.81 \mathrm{~m}$ \\
\hline$D_{p}$ & Maximum interaction range of proximal control & $2.0 \mathrm{~m}$ \\
\hline$D_{a}$ & Maximum interaction range of alignment control & 0.1 \\
\hline$\sigma$ & Amount of noise & $2500 \mathrm{~s}$ \\
\hline$T$ & Duration of experiments & 100 \\
\hline$R$ & Number of runs per setting & \\
\hline
\end{tabular}

Table 1: Parameter values or range of values used in the controller and in simulations. The values in bold are the nominal values, that is, the values used in experiments when the effect of another parameter is explored.

\begin{tabular}{|c|l|c|}
\hline Parameter & Description & Value \\
\hline \hline$N$ & Number of robots & 8 \\
\hline$\rho$ & Proportion of informed robots & 0.25 \\
\hline$T$ & Duration of experiments & $300 \mathrm{~s}$ \\
\hline$R$ & Number of runs per setting & 10 \\
\hline
\end{tabular}

Table 2: Parameter values or range of values used for the real-robots experiments. All the parameters related to the controllers were omitted in this table, since they are the same as in simulation (refer to Table 1).

\subsection{Metrics}

We are interested in having a cohesive swarm of robots that aligns in a common direction in a short time. In the experiments with informed robots, we want this direction to be the desired goal direction, and we want the swarm to travel as far as possible in this direction. We use order (Vicsek et al., 1995) and accuracy (Couzin et al., 2005) as metrics to measure the degree of success in achieving these objectives. Additionally, we also measure the steady-state value that is reached for a given metric and how long it takes for the swarm to reach such value. We call these metrics steady-state value and settling time respectively. Finally, we also measure the effective traveled distance in the desired 
goal direction.

Order: The order metric $\psi$ measures the degree of agreement of the orientations of the robots in the swarm. We first define the vectorial sum of the headings of all $N$ robots as:

$$
\mathbf{b}=\sum_{i=1}^{N} e^{j \theta_{i}}
$$

The order is then defined as:

$$
\psi=\frac{1}{N}\|\mathbf{b}\|
$$

When the robots have a common heading, then $\psi \approx 1$; when they point in different directions, then $\psi \approx 0$.

Accuracy: The accuracy metric $\delta$ is used to measure how close to the goal direction the robots are moving. The accuracy metric is defined in the $[0,1]$ interval in order to be consistent with the order metric and follows the same definition as the one provided in previous flocking studies (Çelikkanat and Şahin, 2010):

$$
\delta=1-\frac{1-\psi \cos (\angle \mathbf{b}-\angle \mathbf{g})}{2}
$$

where $\angle \mathbf{b}$ is the direction of $\mathbf{b}$ and $\angle \mathbf{g}$ is the desired goal direction. Robots with a common heading and also moving in the goal direction will thus have $\delta \approx 1$ and $\psi \approx 1$. Conversely, when $\delta \approx 0$, they are moving in a direction which is opposite to the goal direction, even if they are well aligned. Note that, when the swarm is not aligned, the value of the accuracy is $\delta \approx 0.5$, since $\psi \approx 0$ nullifies the contribution of the cosine term.

Steady-state value: The steady-state value is the asymptotic value reached by either order or accuracy during an experiment. After the system reaches a steady state, we compute the stead-state value as the average value of the metric in the last 100 simulated seconds:

$$
\bar{\mu}=\frac{\sum_{(t=T-100)}^{T} \mu_{t}}{100}
$$

where $\mu_{t}$ denotes either the order $\psi$ or the accuracy $\delta$ at time $t$. 
Settling time: The settling time is the time needed to reach a steady state in order or in accuracy. More precisely, the settling time $t^{*}$ is defined as the time for which $\forall t \geq t^{*}$ we have $\mu_{t} \geq 0.95 \bar{\mu}$. In other words, $t^{*}$ is the time at which and after which the considered metric stays above the $95 \%$ of the steady state of the considered metric $\mu$.

Effective traveled distance: The effective traveled distance is the distance, in meters, traveled in the desired goal direction. It is computed as the total distance traveled projected onto the goal direction $\mathbf{g}$ :

$$
D_{e}=\left(\mathbf{x}_{T}-\mathbf{x}_{0}\right) \cdot \mathbf{g} .
$$

Here, $\mathbf{x}_{T}$ and $\mathbf{x}_{0}$ are the position vectors of the center of mass of the swarm at time $t=T$ and $t=0$, respectively; $\mathbf{g}$ is the goal direction, and $\cdot$ denotes the dot product of two vectors.

\subsection{Experimental setup in simulation}

We compared the proposed MDMC with MIMC, the motion control method used in Turgut et al. (2008a), and in Çelikkanat and Şahin (2010). The experiments can be divided in two sets. In the first set, no robot is informed about any desired goal direction. In the second set, we select a proportion $\rho$ of robots and we inform them about the goal direction g. All the other robots remain uninformed during the entire experiment. The informed robots and the goal direction are randomly selected at the beginning of each run. In all cases, we performed experiments with and without alignment control.

The models described in Section 2 are utilized in the following way. In experiments without informed robots and without alignment control, all robots use Model 2 (Equation 2). In experiments without informed robots and with alignment control, all the robots use Model 4 (Equation 4). In experiments with informed robots and without alignment control, informed robots use Model 3 (Equation 3), whereas the rest of the swarm uses Model 2. Finally, in experiments with informed robots and with alignment control, informed robots use Model 1 (Equation 1) and the rest of the swarm uses Model 4.

We study both the transient and the steady-state behavior of the system. When analyzing the transient behavior, we report the time evolution of the median value of the 
order or of the accuracy metrics, with the corresponding error bars showing the values at the $25 \%$ and the $75 \%$ percentile scores (see Figure 2 for an example). When analyzing the steady-state behavior, we report the box-plot of the distribution of the steady-state values and of the settling times. In experiments with informed robots, we also report the box-plot of the distribution of the effective traveled distances.

In all experiments, we add noise to several components of our system: to the proximal control vector $\mathbf{p}$, to the alignment control vector $\mathbf{a}$, and to the goal direction vector $\mathbf{g}$. We consider noise only in the bearing, as commonly done in flocking studies (Vicsek et al., 1995; Turgut et al., 2008a), and we introduce it by adding to the bearing a random variable homogeneously distributed in the range $(-\sigma 2 \pi,+\sigma 2 \pi)$. For each experimental setting, we execute $R$ independent runs.

At the beginning of each run, $N$ mobile robots are placed within a circle at random positions and orientations. The radius of the circle is chosen so that the mean density of the initial configuration is kept fixed at 5 robots per square meter, indepedently of the number of robots $N$. In the experiments with informed robots or with aligning robots, a light source is also placed at a fixed position in the arena, in such a way to be seen by all robots that use it as a global reference frame.

\subsection{Experimental setup with real robots}

In order to validate the results obtained in simulation with MDMC, we perform experiments on real robots. Eight foot-bots are placed in the arena depicted in Figure 1b. The robots are placed within a circle at the center of the arena at the beginning of each run, each with a random orientation. A light source is placed at the left side of the arena. To measure order and accuracy over time, we built a custom-made tracking system. We place cardboard hats with a directional marker on top of each robot $^{5}$. This marker is detected by an overhead camera placed on one side of the arena, at an height of about 3 meters and pointing towards the floor (Figure $1 \mathrm{~b}$ was taken by this camera). We recorded a movie for each experiment and we analysed each video off-line using the Halcon software ${ }^{6}$. The analysis of each video produced a file containing, for each frame, the orientation of all robots.

\footnotetext{
${ }^{5}$ Note that such hats are used for tracking purposes only and are not detectable by the robots themselves.

${ }^{6} \mathrm{http}: / /$ www.halcon.de/
} 
We conduct two sets of experiments as we did in simulation: without and with informed robots. All parameters are kept the same as in the simulations. We only change the number of robots and the duration of the experiments in order to comply with arena-size constraints. We consider a proportion of $25 \%$ informed robots. The parameters used with real robots are summarized in Table 2. All other parameters are the same as used in simulation (Table 1).

For each of the two sets of experiments, and for each of the two motion control methods, we execute 10 runs and report the median values of the metrics together with their first and third quartiles.

\section{$5 \quad$ Results without informed robots}

In this section, we report results obtained without informed robots, that is, $\mathbf{g}=\mathbf{0}$. We first analyze the transient and the steady-state behavior of the system in simulation, and then we report the results obtained with real robots.

\subsection{Simulations}

Transient behavior: Figure 2 shows the transient behavior of the system in a mediumsized $(N=100)$ and in a large $(N=1000)$ swarm. Figures 2a and 2c show the results obtained without alignment control (Equation 2). As we can see, the swarm reaches an ordered state only when using MDMC. The ordered state is reached within 700 simulated seconds in the medium-sized swarm and within 1500 simulated seconds in large swarms. When MIMC is used, the swarm remains disordered during the entire experiment. Figures $2 \mathrm{~b}$ and $2 \mathrm{~d}$ show the results obtained with alignment control. In this case, the alignment state is reached with both motion control methods, and the response of the system is much faster than without alignment control. The performance of MDMC and MIMC are comparable: the system self-organizes in less than 200 seconds in medium-sized swarms and in less than 400 seconds in large swarms.

Steady state and settling time without using alignment control: Figure 3 shows the steady-state behavior of the system for different swarm sizes. Figure 3a shows box-plots of the distributions of the asymptotic values of order reached without alignment control. 


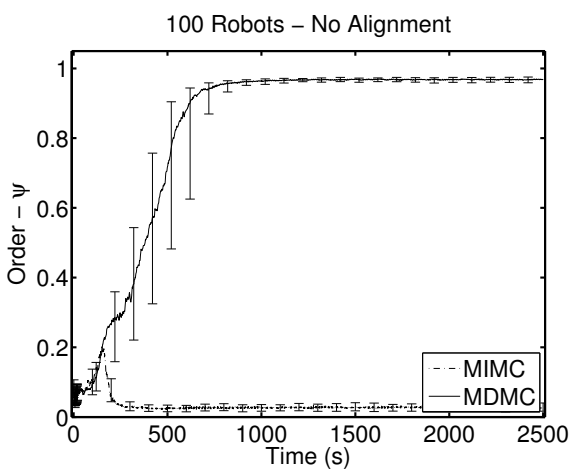

(a)

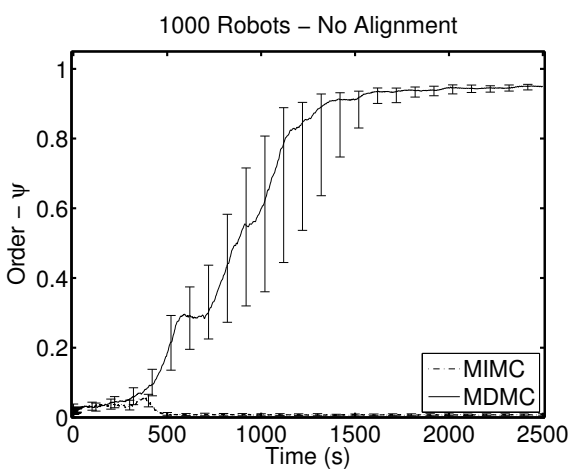

(c)

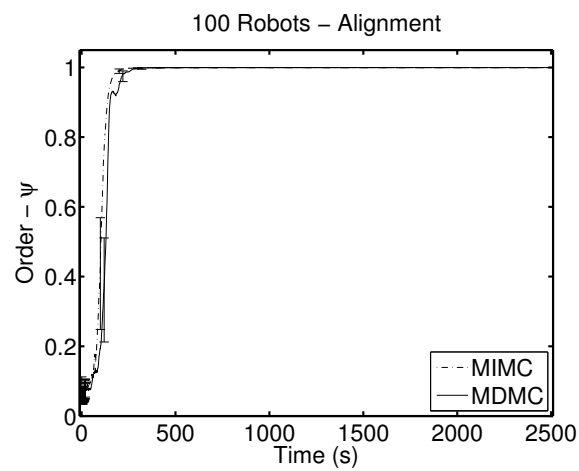

(b)

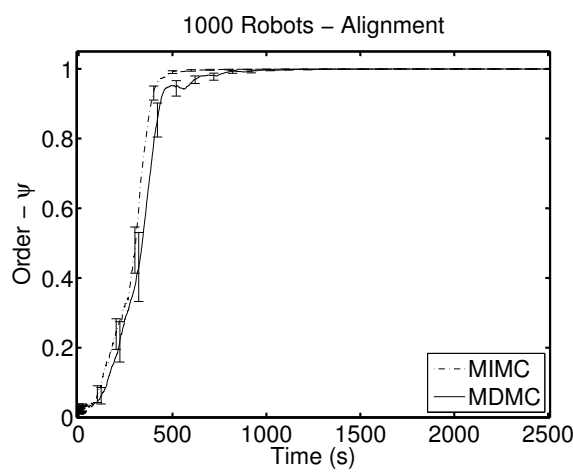

(d)

Figure 2: MDMC vs MIMC in simulation experiments without informed robots. Time evolution of the order metric for (a) 100 robots - without alignment control, (b) 100 robots - with alignment control, (c) 1000 robots - without alignment control and (d) 1000 robots - with alignment control. The line denotes the median of the distribution obtained over $R=100$ runs whereas the error bars represent the interval between the $25 \%$ and the $75 \%$ quartiles of the distribution.

Figure $3 \mathrm{~b}$ shows box-plots of the distributions of the settling times without alignment control.

An important result of this paper is shown in Figure 3a: by using MDMC, the system achieves an ordered state without the need of alignment control for all swarm sizes. The use of MDMC is critical for achieving this result. In fact, as shown in Figure 3a, the system never reaches the ordered state when using MIMC.

Figure 3a also displays points indicating results that were considered as outliers. These are of two different types. Some correspond to cases where the system is trying to selforganize but is too slow to reach its stationary state within the maximum run time of $T=$ $2500 \mathrm{~s}$, and can be easily identified by looking at the time series. The others correspond 


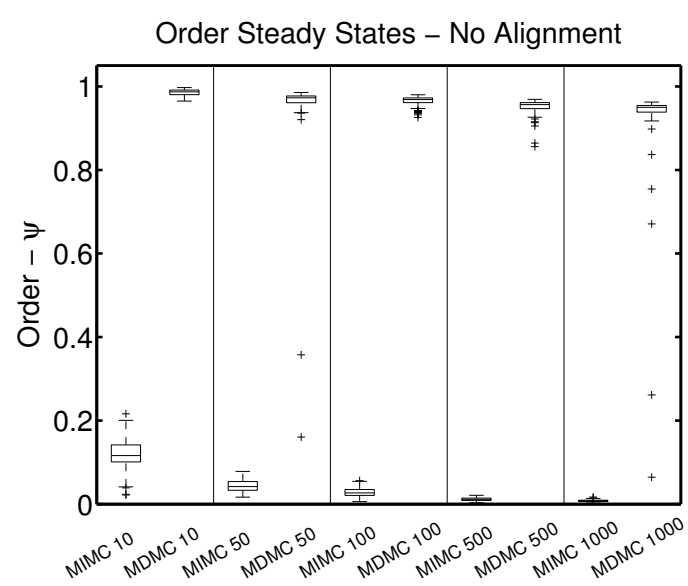

(a)

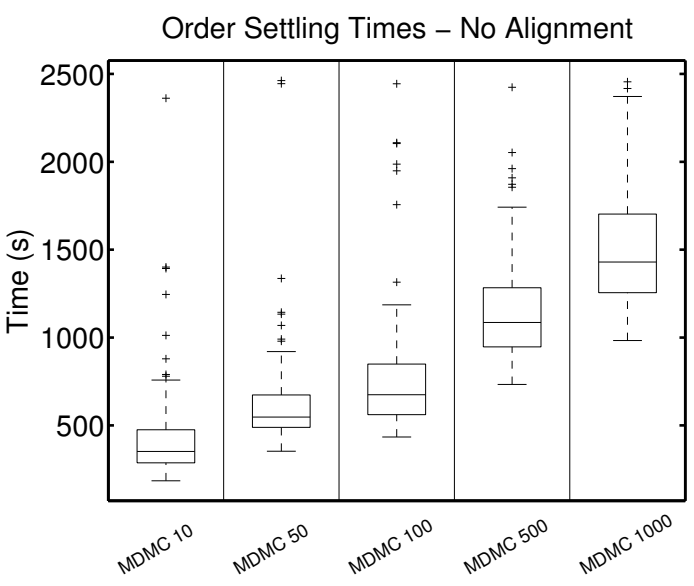

(b)

Figure 3: MDMC vs MIMC in simulations with no informed robots and without alignment control. Box-plots of the distribution of (a) steady-state values and (b) settling times of the order metric for different swarm sizes.

to cases in which the swarm, instead of achieving aligned motion, converged to a rotating state with fixed center of mass, low order $(\psi<0.5)$ and high angular rotation. A video of a rotating state can be found in our supplementary material page (Ferrante et al., 2012a). For the purpose of our current study, we consider these as outliers and focus on the convergence to parallel states. Convergence to a rotating state is rare, becomes less probable if more robots are used, and can be easily suppressed by imposing minor constraints to the robot dynamics. Its analysis goes beyond the scope of this paper and will be presented in a parallel study.

In Figure $3 \mathrm{~b}$ we report the settling time needed to reach the ordered state. The settling time obtained with MDMC increases with increasing swarm sizes, as expected. We also noticed some outliers in the distribution of settling times for MDMC. These correspond to runs where the systems first self-organizes in the rotating solution, and only later, due to noise, reorganizes in the ordered solution. Settling times for MIMC are not reported as the system does not converge to a steady-state in the allotted time (2500 s).

Steady state and settling time when using alignment control: Additional results reported in the supplementary material page (Ferrante et al., 2012a) show that the system reaches the ordered state for any swarm size and any motion control method when alignment control is used. This is consistent with previous studies in flocking (Turgut 


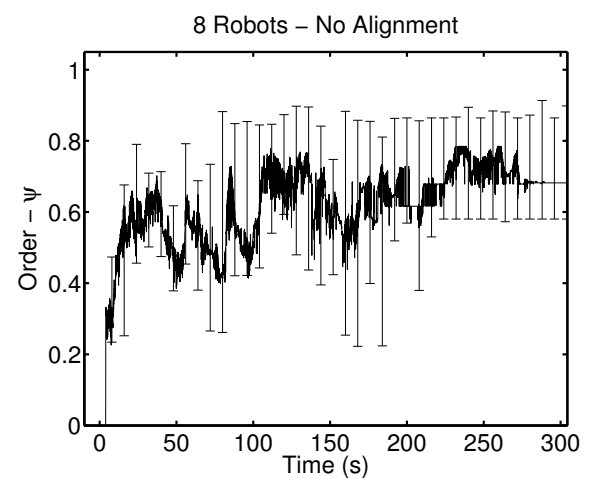

(a)



(b)

Figure 4: Results with no informed robots using MDMC in real-robots experiments. Time evolution of the order metric for 8 robots (a) without alignment control and (b) with alignment control. The line denotes the median of the distribution obtained over $R=10$ runs whereas the error bars span the interval between the $25 \%$ and the $75 \%$ quartiles of the distribution.

et al., 2008a) and, additionally, it shows that MDMC can also be implemented together with alignment control. Additionally, for both methods the medians of the settling times increase with increasing swarm sizes.

\subsection{Real robots}

Figure 4 shows the results obtained with MDMC using real robots. Results in Figure 4a show that, when alignment control is not used, the system reaches reasonable levels of order also with the real robots. The difference in performance when compared to the simulations can be attributed to the different nature and amount of noise on the real robots, which is very difficult to replicate in simulation. Figure $4 \mathrm{~b}$ shows that, when alignment control is used, results are less noisy. This is because alignment control uses a communication device, the RAB, that is noise-free. Its only source of noise comes from orientation measurements via the light sensor, which appears to be very precise. Results with no alignment control also exhibit much larger fluctuations of the order vs time than those with alignment control. Finally, note that both in Figure 4a and in Figure 4b the order becomes constant at $t \approx 280 \mathrm{~s}$ and at $t \approx 180 \mathrm{~s}$, respectively. This is because by time $t \approx 280 \mathrm{~s}$ (respectively $t \approx 180 \mathrm{~s}$ ) all experiments had been stopped due to the robots having reached the borders of the arena. 




(a)

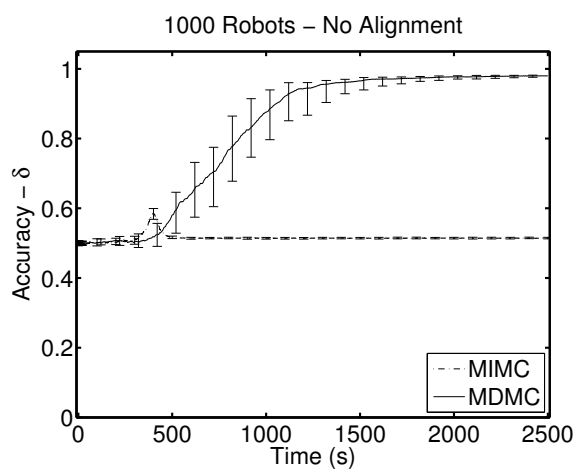

(c)

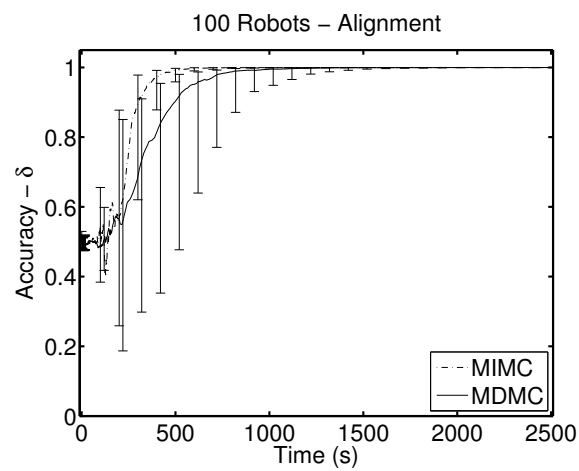

(b)

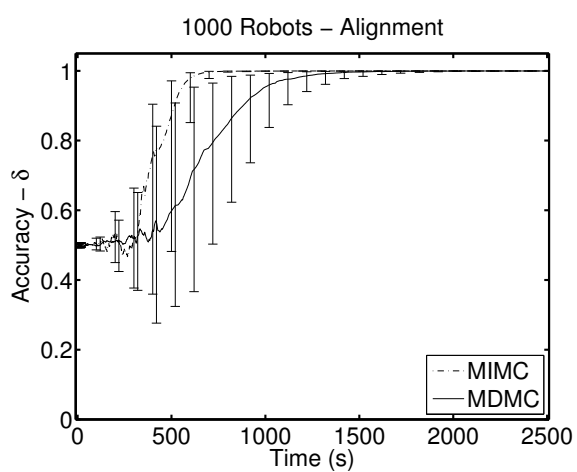

(d)

Figure 5: MDMC vs MIMC in simulations with a proportion of $\rho=0.01$ informed robots. Time evolution of the accuracy metric for (a) 100 robots without alignment control, (b) 100 robots with alignment control, (c) 1000 robots without alignment control and (d) 1000 robots with alignment control. The line denotes the median of the distribution obtained over $R=100$ runs whereas the error bars represent the interval spanned between the $25 \%$ and the $75 \%$ quartiles of the distribution.

\section{$6 \quad$ Results with informed robots}

In this section, we report results obtained with informed robots. Analogously to the experiments in Section 5, we analyze the transient and the steady-state behavior of the system in simulation, and then we report the results obtained with real robots.

\subsection{Simulations}

Transient behavior: Figure 5 shows the transient behavior of the system for $N=100$, $N=1000$ and a proportion of informed robots $\rho=0.01$. Figures $5 \mathrm{a}$ and $5 \mathrm{c}$ show results obtained without alignment control. For this case, the swarm reaches a value of accuracy close to 1 only when using MDMC. This value is reached within $1000 \mathrm{~s}$ and $1500 \mathrm{~s}$ for 


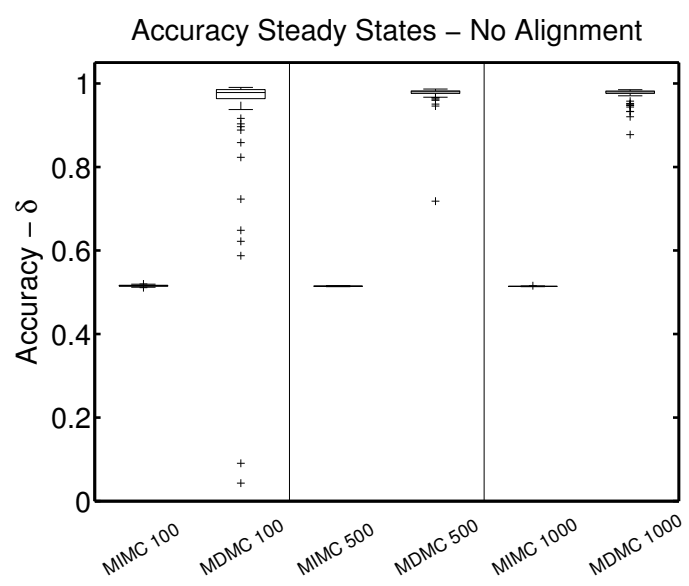

(a)

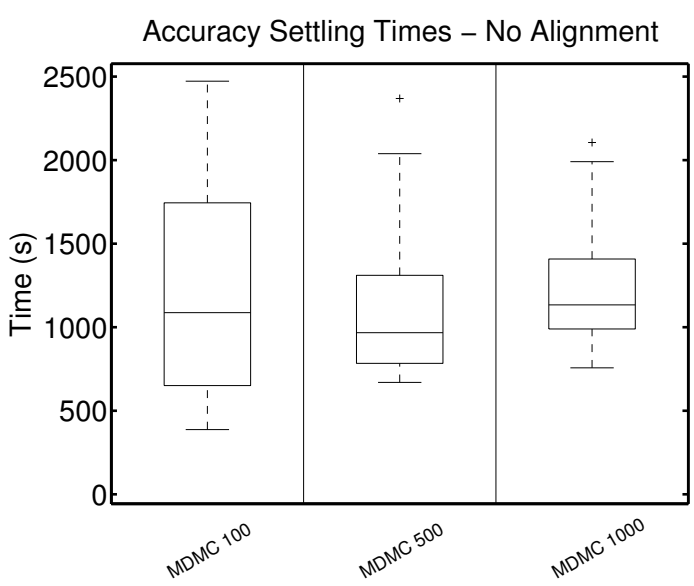

(b)

Figure 6: MDMC vs MIMC in simulations with a proportion of $\rho=0.01$ informed robots without alignment control. Box-plots of the distribution of (a) steady-state values and (b) settling times of the accuracy metric for different swarm sizes.

swarms with $N=100$ and $N=1000$ robots, respectively. When using MIMC, accuracy of the system remains $\delta=0.5$. As explained in Section 4.1, this is the value obtained when the order metric is close to 0 . In other words, when using MIMC without alignment control, the system is not able to reach an ordered state if the proportion of informed robots is small $(\rho=0.01)$. Complete results that include also the value of the order metric are reported in our supplementary material page (Ferrante et al., 2012a). However, as we will show later, if the proportion of informed robots is increased, the system selforganizes also with MIMC. Figures 5b and 5d show the results obtained with alignment control. In this case, a proportion $\rho=0.01$ of informed robots is enough to reach high levels of accuracy for both MIMC and MDMC. Furthermore, MIMC seems to have better performance in terms of convergence time.

\section{Steady state and settling time of accuracy without using alignment control:}

Figure 6 shows the steady-state behavior of the system for different swarm sizes, with $\rho=$ 0.01 and without alignment control. Figures 6 a shows the distribution of the asymptotic value of the accuracy metric. As we can see, MDMC dominates MIMC in all three cases. In facts, the system does not reach an ordered state when MIMC is used. This makes the accuracy metric settle approximately at $\delta \approx 0.5$. In Figure $6 \mathrm{~b}$, we see that the distribution of settling times for MDMC has $1000 \mathrm{~s}$ as median value. 


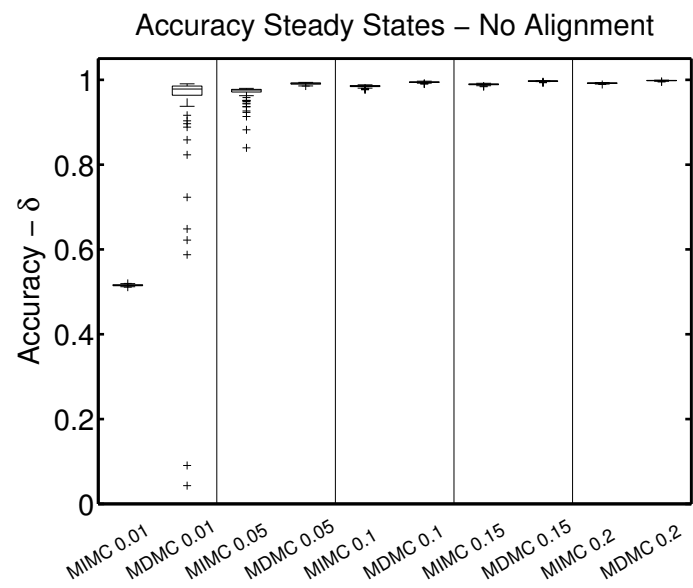

(a)

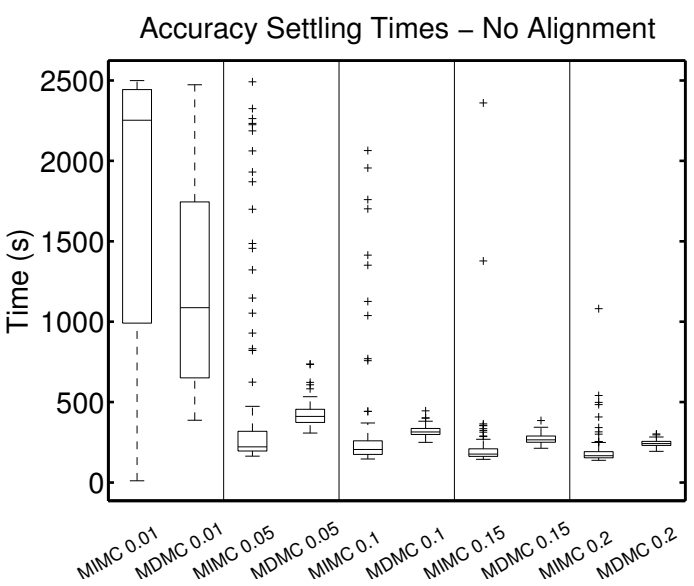

(b)

Figure 7: MDMC vs MIMC in simulations with informed robots, no alignment control, a swarm size of $N=100$ robots and $\rho=\{0.01,0.05,0.1,0.15,0.2\}$. Box-plots of the distribution of (a) steady state values and (b) settling times of the accuracy metric for different proportions of informed robots.

Steady state and settling time of accuracy when using alignment control: Additional results reported in the supplementary material page (Ferrante et al., 2012a) show that, when alignment control is used, the steady-state values of the accuracy are almost always close to 1 . The few outliers that are observed are due to the fact that, in rare cases, the system splits into two groups. The median settling time, as well as its spread, increases with increasing swarm sizes, and increases more with MDMC than with MIMC.

Impact of the proportion of informed robots: Figure 7 reports the results of the study conducted with fixed swarm size $N=100$ and various proportions of informed robots. Figure 7a shows that, when alignment control is not used, MDMC dominates MIMC in terms of accuracy only for $\rho=0.01$. In the other cases, the two methods achieve very similar performance level. However, as shown in Figure 8 and explained below, this apparent equivalence beween MDMC and MIMC in accuracy does not result in an equivalence in the effective traveled distance. Finally, Figure $7 \mathrm{~b}$ shows that the system converges faster with MDMC than with MIMC in the $\rho=0.01$ case, and that the opposite is true for the other cases.

Additional experiments performed with alignment control (Ferrante et al., 2012a) show that the system achieves very good performance levels in all cases. However, MDMC 




(a)

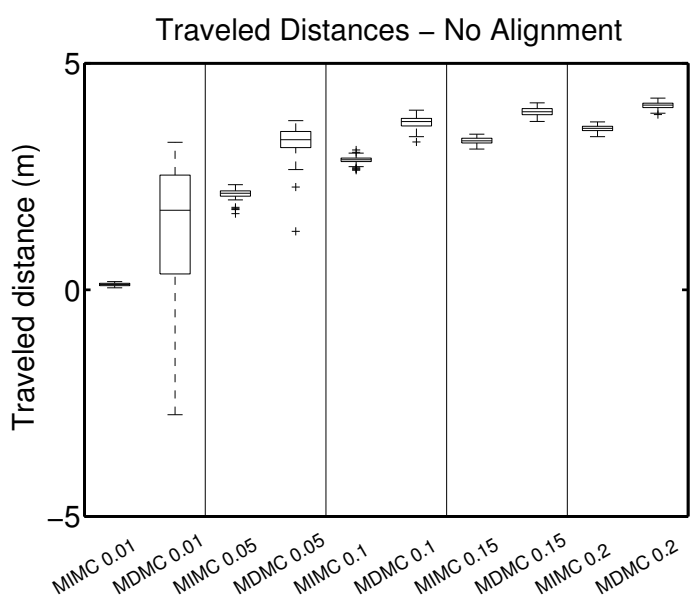

(b)

Figure 8: MDMC vs MIMC in simulations with informed robots and no alignment control. Box-plots of the distribution of effective traveled distance for (a) different swarm sizes $(\rho=0.01)$ and (b) different proportions of informed robots $(N=100)$.

converges slower than MIMC when the proportion of informed robots is low.

Effective traveled distance: Figure 8 shows the box-plots of the distribution of the effective traveled distance for different swarm sizes (fixing $\rho=0.01$ ) and of the proportion of informed robots (fixing $N=100$ ). When alignment control is not used, MDMC consistently outperforms MIMC for any swarm size (Figure 8a). This was already known since, unless when the swarm is small, the system cannot order or achieve high accuracy values when using MIMC. Figure 8b shows that MDMC also consistently outperforms MIMC for any proportion of informed robots. Although the system can self-organize and achieve a high level of accuracy for a larger proportion of informed robots, MDMC still has the advantage of being able to let the swarm travel farther in the desired direction of motion.

Additional experiments performed using alignment control (Ferrante et al., 2012a) show that the swarm is able to travel in the desired goal direction for all swarm sizes. We note that MIMC performs slightly better than MDMC with alignment control, although the latter is still competitive. Finally, we also observe that the two methods perform almost equally for a large proportion of informed robots. 


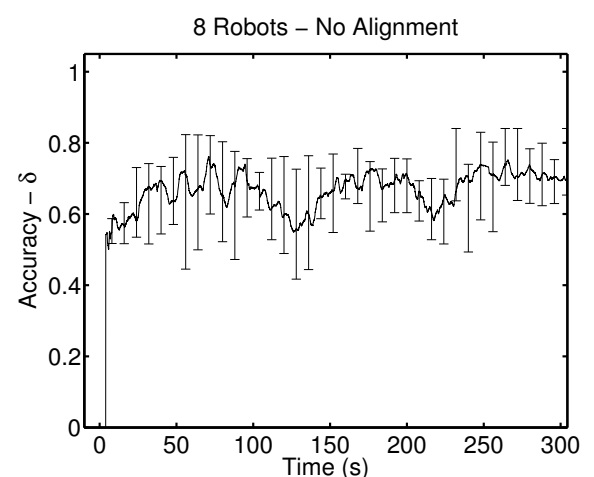

(a)

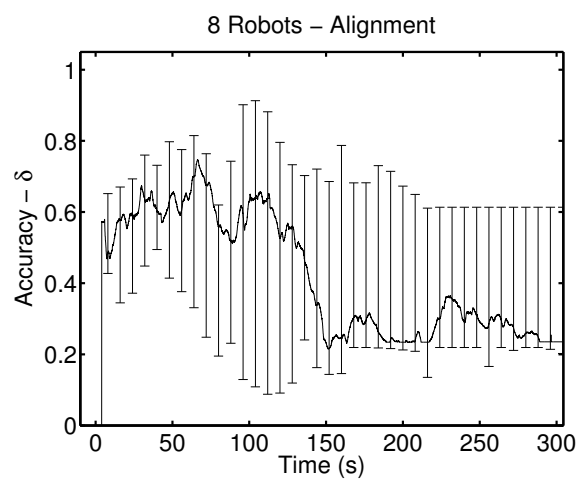

(b)

Figure 9: Results with informed robots using MDMC in real-robots experiments. Time evolution of the accuracy metric for a group of 8 robots and 2 informed robots (a) without alignment control and (b) with alignment control. The line denotes the median of the distribution obtained over $R=10$ runs whereas the error bars represent the interval spanned between the $25 \%$ and the $75 \%$ quartiles of the distribution.

\subsection{Real robots}

Figure 9 shows the results obtained with MDMC using real robots. The results in Figure 9a show that, without alignment control, the system reaches reasonable levels of accuracy. The difference in performance when compared to simulation could be due to the different way in which noise affects the system, since our simulation does not attempt to model realistically the noise. Figure 9b shows that, surprisingly, MDMC performs worse in real robots with alignment control. In fact, in a group of 8 real robots with 2 informed robots, the range and bearing communication device made the swarm agree on a common, random direction very fast. When this happened, the interactions between informed and noninformed robots were not strong enough to let the swarm change its direction in the desired one. Furthermore, the swarm, due to noise, continuously changed its direction, which may explain the increase in the spread and the decrease of the median of the distribution after $t=100 \mathrm{~s}$. By contrast, when alignment control is not used, the swarm never agrees on a common direction. Hence, the movement of the swarm is almost completely driven by the interaction between the informed and the non-informed robots, that in this case is enough to make the swarm move in the desired direction of motion. 


\section{Related work and discussion}

In this section, we review the literature on flocking. We first discuss previous works in robotics, highlighting the novelties of the current paper. We then discuss the most representative work on flocking in statistical physics, comparing the two different perspectives.

\subsection{Robotics}

In robotics, flocking has been studied for the last two decades. We will focus here on works where the method is either directly tested on real robots or at least it has the potential to be readily applied to real robots. We classify the literature according to two categories. In the first, we include works that use alignment control; in the second, those that do not use it.

\subsubsection{Robotics studies with alignment control}

We divide studies where alignment control is used into two categories. In the first, we place studies that relied on external hardware beyond what robots have on-board. In the second, we place studies using on board hardware only.

In works belonging to the first category, authors have either estimated the relative orientation of the robots (Hayes and Dormiani-Tabatabaei, 2002) or emulated an orientation sensing device using an external computer (Holland et al., 2005).

Hayes and Dormiani-Tabatabaei (2002) proposed a flocking method based on collision avoidance and velocity matching where all measurements were emulated via an external computer and broadcast to the robots. Robots compute the position and the velocity of the center of mass of their neighbors, in order to maintain cohesion and to align in the same direction. Furthermore, each robot is informed about the direction to a goal area. Emulated external sensing was also used by Holland et al. (2005), who developed a method based on Reynolds' rules for unmanned aereal vehicles.

In the works that fall within the second category, a local communication unit is always used to implement alignment control. In Campo et al. (2006), robots have to transport an object to a given location. This work is one of the pioneering studies of coordinated motion with purely on-board local communication, implemented via an LED ring and an omni-directional camera. 
Turgut et al. (2008a) proposed a method based on proximal control and alignment control that achieves ordered motion in a random direction. Proximal control and alignment control ware implemented using proximity sensors and a virtual heading sensor (VHS), respectively. The VHS combines a digital compass and a communication unit: each robot measures its orientation and broadcasts it. In this way, the orientation of a robot is sensed "virtually" by its neighbors. In a follow-up study, Gökçe and Şahin (2010) introduced a goal-following behavior and studied the effect that a noisy goal direction has on the swarm motion. Çelikkanat and Şahin (2010), inspired by the work of Couzin et al. (2005), provided a goal direction to some of the robots and showed that these are enough to guide a large swarm.

Ferrante et al. (2010) proposed a communication strategy that uses the same range and bearing sensor considered in this paper. The authors achieved good flocking performance in environments where the goal direction changes over time. Stranieri et al. (2011) studied self-organized flocking in a heterogeneous swarm of robots, where some of the robots use alignment control, and the rest of the swarm only uses proximal control. Under these conditions, the swarm can still organize and move in a common random direction. Recently, in Ferrante et al. (2012b), a self-adaptive communication strategy was proposed to deal with flocking in situations with two conflicting goal directions with different priorities. The authors showed that the swarm can always follow the highest priority goal direction without splitting.

\subsubsection{Robotics studies without alignment control}

In this category, we place studies that do not use alignment control. Instead, alignment to a given direction is typically induced by introducing a large majority of robots that are informed about a goal direction (Matarić, 1994) or light-source direction (Spears et al., 2004).

Matarić (1994) proposed a flocking method based on a set of "basis behaviors": safewandering, aggregation, dispersion and goal-following. The robots sense obstacles in the environment, localize themselves with respect to a set of stationary beacons and broadcast their position. Robots moved cohesively in a goal direction known to all robots.

Kelly and Keating (1996) proposed a flocking method based on a leader-following 
behavior. They used an active infra-red sensing system to sense the range and bearing of other robots and a radio-frequency system for dynamically electing the leader. Multiple informed robots could co-exist in the swarm and, in their presence, the swarm was able to split to overcome obstacles.

Baldassarre et al. (2003) used artificial evolution to evolve a flocking behavior with a group of four simulated robots. The robots were equipped with proximity and light sensors. They used the former to perceive each others' relative position and orientation, and used the latter to perceive a common goal direction.

Nembrini et al. (2002) proposed a minimalistic method to achieve flocking using only a local communication device and a sensor able to detect obstacles and beacons. Some robots were informed about a goal direction and signaled their status using their beacon. They achieved a swarming behavior where robots dynamically disconnect and reconnect to the swarm.

Spears et al. (2004) proposed a flocking method based on attraction/repulsion and viscous forces. The robots form a regular lattice structure using the range and bearing of their neighbors and move in a goal direction given by a light source perceived by the majority of the robots.

Moslinger et al. (2009) proposed a method for minimalistic flocking based on attraction and repulsion zones with different threshold levels. By adjusting these levels, they achieved flocking with a small group of robots in a constrained environment. In this work, as in our study, no robots used neither goal direction nor alignment information. However, the flocking behavior was limited since the group could not stay cohesive all the time.

Monteiro and Bicho (2010) developed a control method based on leader-follower dynamics to move a swarm in formation towards a target. The location of the target is known to some informed robots, assumed to be identifiable within the swarm.

Recently, Tarcai et al. (2011) studied a system composed of very simple remotecontrolled (RC) boats subject to inelastic, natural collisions between each other. They placed the boats in a toroidal pool and observed that the swarm organizes in a clockwise or counter-clockwise motion after a certain amount of time. They also studied the effect of adding informed boats into the system. This work can be considered of hybrid nature, between robotics and statistical physics, but is only analyzed here. 


\subsubsection{Discussion}

The main contribution of this paper is to propose a motion control method that achieves flocking without alignment control and without informed robots. Therefore, the unique characteristic of this work with respect to the literature analyzed above is that we explicitily study motion control, which has received little or no attention at all.

Since our method can achieve flocking even in absence of alignment control, it is unique when compared to the literature dicussed in Section 7.1.1. When comparing to the studies discussed in Section 7.1.2 where alignment control was not used, our study presents the following differences. Our analysis considers only a few informed individuals or no informed individuals, while almost all of the previous studies (Matarić, 1994; Kelly and Keating, 1996; Baldassarre et al., 2003; Nembrini et al., 2002; Spears et al., 2004; Monteiro and Bicho, 2010) included a large proportion of informed robots, to help the system reach an ordered state. The rest of the studies either achieved sub-optimal performance in swarm cohesion when compared to our work (Moslinger et al., 2009) (the swarm was splitting and rejoining very often in a bounded arena) or analyzed a system under very specific environmental conditions (Tarcai et al., 2011). To the best of our knowledge, our work is the first to achieve both ordered and cohesive flocking performance in an environment with no constraints on the boundary conditions.

\section{2 $\quad$ Statistical physics}

In statistical physics, flocking is studied with the aim of searching for a theory of selfpropelled collective motion applicable to a wide range of systems. Often, the approach is to consider simple models, such as point-particle systems in which actuation or sensing noise plays a role similar to that of temperature in standard physical systems. The resulting dynamics are analyzed using tools from non-equilibrium statistical physics.

Here, we describe briefly some of the most representative statistical physics flocking studies. We classify them into two groups: with and without explicit alignment rules. A complete survey can be found in (Vicsek and Zafiris, 2010). 


\subsubsection{Statistical physics models with alignment}

The first flocking model developed within statistical physics was SDP (self-driven particles) (Vicsek et al., 1995). In SDP, particles advance at constant speed and use an alignment rule: at every time-step, each particle's orientation is set to the average orientation of its neighbors plus a noise component. The model includes neither attraction nor repulsion. The authors found that a phase transition separates the ordered state (particles moving in a common direction) from the disordered state (particles with random orientation). The transition is reached by either varying the amount of noise or the particle density.

Gregoire et al. (2003) extended the SDP model by adding the attraction and repulsion rules. Noise is introduced by adding a small randomly oriented vector to the particles' heading. As in the Vicsek et al. (1995) case, the authors observed a transition between an ordered and a disordered state at a critical noise value. The details of this transition appear to be different, and this issue is still a matter of debate.

Aldana and Huepe (2003) considered the vectorial network model (VNM), in which individuals do not move in space but are nodes of a network. Each node has an associated orientation that evolves following an SDP model where neighbors are nodes directly connected through a link. The authors found that an order-disorder transition equivalent to that in the SDP model could be obtained only when long-range interactions are present. The VNM was extended by Turgut et al. (2008b) to analyze the self-organized flocking of robots that follow the method proposed in Turgut et al. (2008a).

Finally, Cucker and Huepe (2008) introduced informed individuals and studied theoretically the behavior of the swarm. They considered a Laplacian model, where each particle tries to minimize the difference between its velocity and that of its neighbors, that are defined according to an adjacency matrix.

\subsubsection{Statistical physics models without alignment}

Szabó et al. (2006) proposed one of the first models that achieves ordered motion using locally interacting particles and no explicit alignment rule. The goal was to fit experimental data they gathered on the dynamics of collectively migrating tissue cells. The authors observed an order-disorder phase transition occurring at critical mean density of 
particles. Their model has many similarities with MDMC. For example, particles interact through attraction-repulsion forces and move forward with non-constant speed. An important difference, however, is that in their model each particle is not constrained to only translate parallel to its orientation but can also advance sideways. Due to this difference, their model is not directly applicable to standard non-honolomic mobile robots, which are constrained in their movements by their wheels. Similar results to those of Szabó et al.'s were also obtained by Erdmann and Mikhailov (2005), who considered particle motion in two dimensions and later extended the model to three dimensions (Strefler et al., 2008).

Grossman et al. (2008) considered a system composed of particles that interact only through inelastic collisions on a $2 \mathrm{D}$ plane with reflecting circular boundary conditions. They observed complex phenomena such as ordered motion, vortices and chaos. They then studied the system in elliptic-shaped arenas, showing that more complex dynamical patterns, such as particles moving together in sub-groups, could be observed.

In Romanczuk et al. (2009), the authors considered particles with asymmetric nonisotropic interactions. Their model represents "escape-pursuit" dynamics. A particle is attracted to particles in the front (pursuit) and is repelled from particles on the back (escape). Here again, the authors find ordered phases displaying collective motion for high levels of the mean density. At low densities, collective motion is only achieved for specific combinations of the parameters that determine the escape-pursuit dynamics.

\subsubsection{Discussion}

The above review allows us to speculate on the key mechanisms required for a method to make a swarm self-organize to a flocking state with no alignment rule and informed robots. For this to happen, we have observed in simulation videos (Ferrante et al., 2012a) that robots must first oscillate in place while turning to adjust their orientation. This process produces small coherent aligned regions that gradually grow until they span the whole group, which then starts moving. We have observed that, in cases where the system fails to self-organize, robots oscillate and turn in place, without creating coherently moving regions. By combining these observations with our survey of successful and unsuccessful methods, we formulate here hypotheses on the conditions required for a method to achieve collective motion. 
A first component that we believe is required to reach an ordered flocking state is continuous angular dynamics. In most statistical physics models, the orientation relaxes in a single time-step to the direction dictated by the model, leading to discrete jumps from one angle to the next. These jumps often leave robots pointing in directions that are still not fully coherent with their neighbors', which may have switched to a very different heading on the same time-step. Instead, in models that successfully order (such as MDMC and Szabó et al. (2006)) each robot adjusts its angular velocity (and not its angle) at each time-step, while the angle relaxes in a continuous manner. These smooth dynamics allow the system to gradually reconfigure as it converges towards the ordered state.

Another component we believe is essential is the dampening of the rotational and translational dynamics as the robot approaches a stable, locally ordered state. In MIMC, this condition is not satisfied, and robots close to a stable local configuration still have high angular velocities, as the angle between the flocking vector and the orientation vector is never zero. A similar situation arises if the forward component of the robot speed is made constant by setting $K_{1}=0$ in the definition of the MDMC model. In this case, high relative velocities between neighbors remain possible when close to a stable configuration.

The two conditions described above are satisfied by MDMC and Szabó et al. (2006). However, a rigorous confirmation of whether these conditions are indeed necessary can only be achieved through a detailed theoretical study, which is currently ongoing.

\section{Conclusions and future work}

In this paper, we have presented a novel motion control method to achieve flocking that can be implemented on robots with limited hardware requirements. Only the relative distance and bearing to neighboring robots are needed to execute the attraction-repulsion dynamics that leads to flocking. Furthermore, to implement MDMC robots do not need to sense each others orientation to perform alignment control, as required by the Reynolds model. The proposed method could thus make possible, in the future, the production of a very large swarm of robots able to perform flocking at a substantially lower cost due to the relatively simple hardware required.

We performed experiments, using both a realistic simulator and real robots. We showed that, with MDMC, self-organized collective motion in a random direction can be achieved. 
Also, MDMC can be implemented including informed robots that have a desired goal direction to be followed, and only a small proportion of such robots is needed to lead a large swarm in the goal direction. Furthermore, MDMC without alignment control and with informed robots allows the swarm to travel farther in the goal direction, when compared to the MIMC method, that was used as a reference. Finally, we carried out a systematic study where we measured and compared the performance of the two methods in various settings: with and without alignment control and with and without informed robots.

This work presents several opportunities for future studies and/or applications. From a theoretical standpoint, we are conducting a more rigorous analysis of the proposed motion control method. We are studying why the proposed model has the properties shown here while others do not. We are also analyzing under which conditions the swarm exhibits the rotating motion on the spot that was mentioned in the results section.

From the point of view of possible applications, the method could be applied to different types of robots, requiring only relatively simple hardware.

An additional possible research direction would be to extend the proposed method to flocking in three dimensional space. These results could be applied to aerial and submarine swarms, where often communication and long range sensing are very difficult to achieve. 


\section{Acknowledgments}

This work was partially supported by the European Union through the ERC Advanced Grant "E-SWARM: Engineering Swarm Intelligence Systems" (contract 246939) and the Future and Emerging Technologies project ASCENS and by the Vlaanderen Research Foundation Flanders (Flemish Community of Belgium) through the H2Swarm project. Cristián Huepe acknowledges support from the US National Science Foundation (Grant No. PHY-0848755). Marco Dorigo acknowledges support from the F.R.S.-FNRS of Belgium's French Community, of which he is a Research Director.

\section{References}

M. Aldana and C. Huepe. Phase transitions in self-driven many-particle systems and related non-equilibrium models: a network approach. Journal of Statistical Physics, 112(1-2):135-153, 2003.

G. Baldassarre, S. Nolfi, and D. Parisi. Evolving mobile robots able to display collective behaviors. Artificial Life, 9(3):255-267, 2003.

M. Bonani, V. Longchamp, S. Magnenat, P. Rétornaz, D. Burnier, G. Roulet, F. Vaussard, H. Bleuler, and F. Mondada. The MarXbot, a miniature mobile robot opening new perspectives for the collective-robotic research. In 2010 IEEE/RSJ International Conference on Intelligent Robots and Systems (IROS 2010), pages 4187-4193, Piscataway, NJ, 2010. IEEE Press.

A. Campo, S. Nouyan, M. Birattari, R. Groß, and M. Dorigo. Negotiation of goal direction for cooperative transport. In M. Dorigo, L. M. Gambardella, M. Birattari, A. Martinoli, R. Poli, and T. Stützle, editors, Ant Colony Optimization and Swarm Intelligence (ANTS) 2006, volume 4150 of Lecture Notes in Computer Science, pages 191-202, Berlin, Germany, 2006. Springer.

H. Çelikkanat and E. Şahin. Steering self-organized robot flocks through externally guided individuals. Neural Computing and Applications, 19(6):849-865, 2010.

I. Couzin, J. Krause, R. James, G. Ruxton, and N. Franks. Collective Memory and Spatial Sorting in Animal Groups. Journal of Theoretical Biology, 218(1):1-11, 2002. 
I. D. Couzin, J. Krause, N. R. Franks, and S. A. Levin. Effective leadership and decisionmaking in animal groups on the move. Nature, 433:513-516, 2005. doi: 10.1038/nature03236.

F. Cucker and C. Huepe. Flocking with informed agents. MathematicS in Action, 1:1-25, 2008.

M. Dorigo, D. Floreano, L. M. Gambardella, F. Mondada, S. Nolfi, T. Baaboura, M. Birattari, M. Bonani, M. Brambilla, A. Brutschy, D. Burnier, A. Campo, A. L. Christensen, A. Decugnière, G. Di Caro, F. Ducatelle, E. Ferrante, A. Förster, J. Guzzi, V. Longchamp, S. Magnenat, J. Martinez Gonzales, N. Mathews, M. Montes de Oca, R. O'Grady, C. Pinciroli, G. Pini, P. Rétornaz, J. Roberts, V. Sperati, T. Stirling, A. Stranieri, T. Stützle, V. Trianni, E. Tuci, A. E. Turgut, and F. Vaussard. Swarmanoid: a novel concept for the study of heterogeneous robotic swarms. IEEE Robotics $\mathscr{E}$ Automation Magazine, in press.

U. Erdmann and A. Mikhailov. Noise-induced transition from translational to rotational motion of swarms. Physical Review E, 71(051904):1-7, 2005.

E. Ferrante, A. E. Turgut, N. Mathews, M. Birattari, and M. Dorigo. Flocking in stationary and non-stationary environments: A novel communication strategy for heading alignment. In R. Schaefer, C. Cotta, J. Kołodziej, and G. Rudolph, editors, Parallel Problem Solving from Nature - PPSN XI, volume 6239 of Lecture Notes in Computer Science, pages 331-340, Berlin, Germany, 2010. Springer.

E. Ferrante, A. E. Turgut, C. Huepe, A. Stranieri, C. Pinciroli, and M. Dorigo. Self-organized flocking with a mobile robot swarm: a novel motion control method. Complete data, 2012a. Supplementary information page at http://iridia.ulb.ac.be/supp/IridiaSupp2012-007/.

E. Ferrante, A. E. Turgut, A. Stranieri, C. Pinciroli, M. Birattari, and M. Dorigo. A self-adaptive communication strategy for flocking in stationary and non-stationary environments, 2012b. Technical report/IRIDIA/2012-002. IRIDIA, Université Libre de Bruxelles, Belgium. 
F. Gökçe and E. Şahin. The pros and cons of flocking in the long-range "migration" of mobile robot swarms. Theoretical Computer Science, 411(21):2140-2154, 2010.

G. Gregoire, H. Chate, and Y. Tu. Moving and staying together without a leader. Physica D, 181(3-4):157-170, 2003.

D. Grossman, I. Aranson, and E. Jacob. Emergence of agent swarm migration and vortex formation through inelastic collisions. New Journal of Physics, 10(023036):1-11, 2008.

A. Hayes and P. Dormiani-Tabatabaei. Self-organized flocking with agent failure: Off-line optimization and demonstration with real robots. In Proc. of the IEEE Int. Conf. on Robotics and Automation, pages 3900-3905, Piscataway, NJ, 2002. IEEE Press.

S. Hettiarachchi and W. Spears. Distributed adaptive swarm for obstacle avoidance. International Journal of Intelligent Computing and Cybernetics, 2(4):644-671, 2009.

O. Holland, J. Woods, R. Nardi, and A. Clark. Beyond swarm intelligence: the ultraswarm. In Proc. of the IEEE Swarm Intelligence Symposium, pages 217-224, Piscataway, NJ, 2005. IEEE Press.

I. Kelly and D. Keating. Flocking by the fusion of sonar and active infrared sensors on physical autonomous robots. In Proc. of the Third Int. Conf. on Mechatronics and Machine Vision in Practice, pages 14-17, Piscataway, NJ, 1996. IEEE Press.

M. J. Matarić. Interaction and Intelligent Behavior. PhD thesis, MIT, MA, 1994.

S. Monteiro and E. Bicho. Attractor dynamics approach to formation control: theory and application. Autonomous Robots, 29(3-4):331-355, 2010.

C. Moslinger, T. Schmickl, and K. Crailsheim. A minimalistic flocking algorithm for swarm robots. In G. Kampis and E. Szathmáry, editors, European Conference of Artificial Life (ECAL 2009), pages 375-382, Berlin, Germany, 2009. Springer.

J. Nembrini, A. F. T. Winfield, and C. Melhuish. Minimalist coherent swarming of wireless networked autonomous mobile robots. In J. Hallam, editor, From Animals to Animats, volume 7, pages 373-382, Cambridge, MA, 2002. MIT Press. 
C. Pinciroli, V. Trianni, R. O'Grady, G. Pini, A. Brutschy, M. Brambilla, N. Mathews, E. Ferrante, G. Di Caro, F. Ducatelle, T. Stirling, A. Gutiérrez, L. M. Gambardella, and M. Dorigo. ARGoS: a modular, multi-engine simulator for heterogeneous swarm robotics. In Proceedings of the IEEE/RSJ International Conference on Intelligent Robots and Systems (IROS 2011), pages 5027-5034. IEEE Computer Society Press, Los Alamitos, CA, 2011.

C. Reynolds. Flocks, herds and schools: A distributed behavioral model. In M. C. Stone, editor, SIGGRAPH '87: Proc. of the 14th annual conference on computer graphics and interactive techniques, pages 25-34, New York, 1987. ACM Press.

P. Romanczuk, I. Couzin, and L.Schimansky-Geier. Collective motion due to individual escape and pursuit response. Physical Review Letters, 102(010602):1-4, 2009.

S. J. Simpson, G. A. Sword, P. D. Lorch, and I. D. Couzin. Cannibal crickets on a forced march for protein and salt. Proceedings of the National Academy of Sciences, USA, 103 (11):4152-4156, 2006.

W. M. Spears, D. F. Spears, J. C. Hamann, and R. Heil. Distributed, physics-based control of swarms of vehicles. Autonomous Robots, 17:137-162, 2004.

A. Stranieri, E. Ferrante, A. E. Turgut, V. Trianni, C. Pinciroli, M. Birattari, and M. Dorigo. Self-Organized flocking with a heterogeneous mobile robot swarm. In Proceedings of the European Conference on Artificial Life 2001, pages 789-796, Cambridge, MA, 2011. MIT Press.

J. Strefler, U. Erdmann, and L. Schimansky-Geier. Swarming in three dimensions. Physical Review E, 78(031927):1-8, 2008.

R. Suzuki and S. Sakai. Movement of a group of animals. Biophysics, 13(281-282), 1973.

B. Szabó, G. Szöllösi, B. Gönci, Z. Jurànyi, D. Selmeczi, and T. Vicsek. Phase transition in the collective migration of tissue cells: Experiment and model. Physical Review E, 74(061908):1-5, 2006.

N. Tarcai, C. Virgh, D. bel, M. Nagy, P. L. Vrkonyi, G. Vsrhelyi, and T. Vicsek. Patterns, transitions and the role of leaders in the collective dynamics of a simple robotic flock. 
Journal of Statistical Mechanics: Theory and Experiment, 2011(04):P04010, 2011. doi: 10.1088/1742-5468/2011/04/P04010.

A. E. Turgut, H. Çelikkanat, F. Gökçe, and E. Şahin. Self-organized flocking in mobile robot swarms. Swarm Intelligence, 2(2):97-120, 2008a.

A. E. Turgut, C. Huepe, H. Çelikkanat, F. Gökçe, and E. Şahin. Modeling phase transition in self-organized mobile robot flocks, volume 5217 of Lecture Notes in Computer Science, pages 108-119. Springer, Berlin, Germany, 2008b.

T. Vicsek and A. Zafiris. Collective motion, 2010. arXiv:1010.5017v2.

T. Vicsek, A. Czirok, E. Ben-Jacob, I. Cohen, and O. Shochet. Novel type of phase transition in a system of self-driven particles. Physical Review Letters, 75(6):1226-1229, 1995.

G. Voronoi. Nouvelles applications des paramètres continus à la théorie des formes quadratiques. Journal für die Reine und Angewandte Mathematik, 133(97-178):198-287, 1908. 


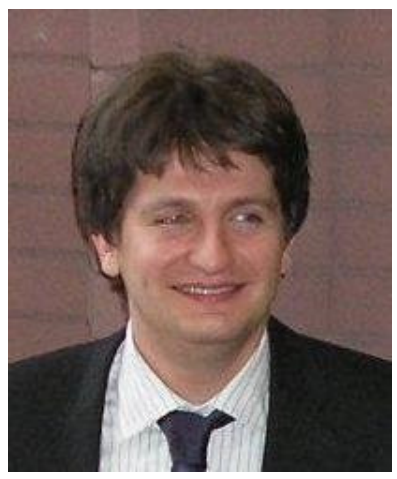

Eliseo Ferrante has received a B.Sc. in Computer Science Engineering from Politecnico di Milano in 2004, Italy, a M.Sc. in Computer Science Engineering from Politecnico di Milano in 2007, Italy, and a M.Sc. in Computer Science from University of Illinois at Chicago, USA, in 2007. He is working as a Research Associate at the Dept. of Biology at KULeuven, Belgium, and is also a Ph.D. student at Université Libre de Bruxelles, Belgium, under the supervision of prof. Marco Dorigo.

E-mail: eliseo.ferrante@bio.kuleuven.be

Address: Laboratory of Socioecology and Social Evolution

Naamsestraat 59, B-3000 Leuven, Belgium 




Ali Emre Turgut has received a B. Sc. in Mechanical Engineering from Middle East Technical University in 1996, Turkey, a M. Sc. in Mechanical Engineering from Middle East Technical University in 2000, Turkey, and Ph.D. in Mechanical Engineering from Middle East Technical University at Kovan Research Laboratory in 2008, Turkey. He worked as a post-doctoral researcher at Université Libre de Bruxelles, IRIDIA, Belgium and as a research associate at the Department of Biology at KULeuven, Belgium during 2008-2012.

E-mail: aturgut@iridia.ulb.ac.be

Address: IRIDIA, CoDE, Université Libre de Bruxelles, 50 Av. Franklin Roosevelt CP 194/6, B-1050 Brussels, Belgium 


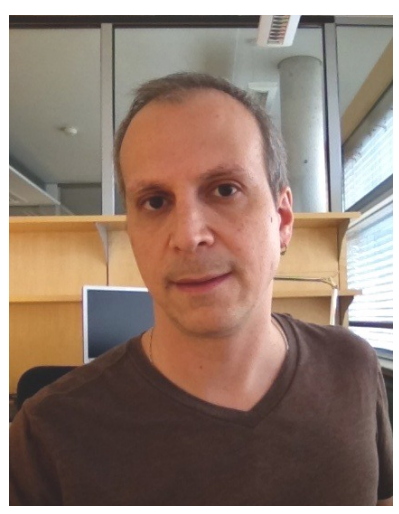

Cristián Huepe is an unaffiliated research scientist based in Chicago and a visiting scholar at the Northwestern University Institute on Complex Systems (NICO). He received his Ph.D. in Physics from University of Paris 7 and École Normale Supérieure de Paris in 1999 and his B.Sc. in Physics from Universidad de Chile in 1994. He was a postdoctoral researcher at University of Chicago from 2000 to 2002 and at Northwestern University from 2002 to 2005. His research has spanned various fields in nonlinear dynamics, statistical physics and complex systems, including superfluidity, Bose-Einstein condensation, complex networks and collective motion. In 2006 he started CHuepe Labs Inc., an independent research organization that has been funded by grants from the US National Science Foundation.

E-mail: cristian@northwestern.edu

Address: 614 N Paulina St, Chicago IL 60622, USA 


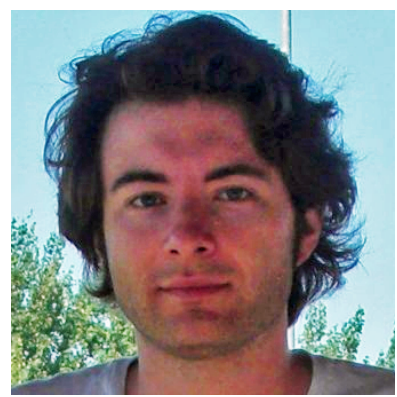

Alessandro Stranieri has received a M.Sc. in Computer Engineering from Politecnico di Milano (Italy) in 2009. During his Master he focused his studies on Artificial Intelligence and Robotics. Since 2010, he is pursuing his Ph.D. at the IRIDIA laboratory of the Université Libre de Bruxelles (Belgium) under the supervision of Prof. Marco Dorigo. The main area of his research is Swarm Robotics and in particular he focuses on the use of vision in multi-robot systems for exploration and environment mapping.

E-mail: alessandro.stranieri@ulb.ac.be

Address: IRIDIA, CoDE, Université Libre de Bruxelles, 50 Av. Franklin Roosevelt CP 194/6, B-1050 Brussels, Belgium 


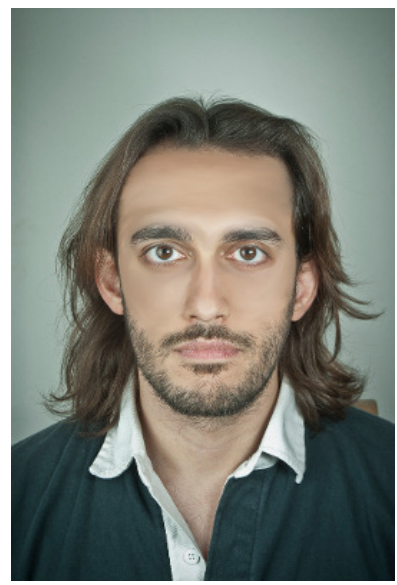

Carlo Pinciroli is a Ph.D. student at IRIDIA, CoDE, Université Libre de Bruxelles in Belgium. Before joining IRIDIA, in 2005 he obtained a Master's degree in Computer Engineering at Politecnico di Milano, Milan, Italy and a second Master's degree in Computer Science at University of Illinois at Chicago, IL, USA. In 2007 he also obtained a Diplôme d'études approfondiés from the Université Libre de Bruxelles. The focus of his research is computer simulation and swarm robotics.

E-mail: cpinciro@ulb.ac.be Address: IRIDIA, CoDE, Université Libre de Bruxelles, 50 Av. Franklin Roosevelt CP 194/6, B-1050 Brussels, Belgium 


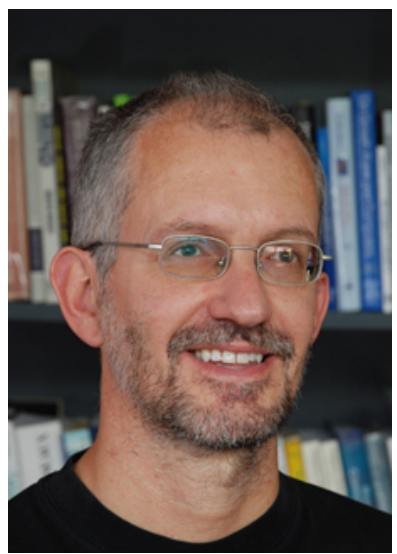

Marco Dorigo is a research director of the F.R.S-FNRS, the Belgian National Fund for Scientific Research, and of IRIDIA, the artificial intelligence laboratory of the Université Libre de Bruxelles. He is the inventor of the ant colony optimization metaheuristic. His current research interests include swarm intelligence and swarm robotics. Dr. Dorigo is the Editor-in-Chief of Swarm Intelligence, and an Associate Editor or member of the Editorial Board of many journals on computational intelligence and adaptive systems. He is a Fellow of the IEEE and of the ECCAI. In 1996 he was awarded the Italian Prize for Artificial Intelligence, in 2003 the Marie Curie Excellence Award, in 2005 the Dr A. De Leeuw-Damry-Bourlart award in applied sciences, in 2007 the Cajastur International Prize for Soft Computing, and in 2010 an ERC Advanced Grant.

E-mail: mdorigo@ulb.ac.be

Address: IRIDIA, CoDE, Université Libre de Bruxelles, 50 Av. Franklin Roosevelt CP 194/6, B-1050 Brussels, Belgium 